\title{
Sopravvivenza di bambini trattati con sebelipase alfa per deficit di lipasi acida lisosomiale: uno studio aperto, multicentrico, a dosi crescenti di farmaco
}

\author{
Salvatore Cannavò ${ }^{1}$
}

Pubblicato online: 20 novembre 2017

(C) Springer International Publishing AG, part of Springer Nature 2017

\section{Commento a:}

Survival in infants treated with sebelipase alfa for lysosomal acid lipase deficiency: an open-label, multicenter, dose-escalation study.

S.A. Jones, S. Rojas-Caro, A.G. Quinn, M. Friedman, S. Marulkar, F. Exgu, O. Zaki, J.J. Gargus, J. Hughes, D. Plantaz, R. Vara, S. Eckert, J.B. Arnoux, A. Brassier, K.H. Le Quan Sang, V. Valayannopoulos.

Orphanet J Rare Dis (2017) 12:25

Il deficit di lipasi acida lisosomiale (LAL-D) causa una rara malattia endocrino-metabolica per l'accumulo di esteri del colesterolo e trigliceridi soprattutto nel fegato, nella milza, nei surreni, nei muscoli, nella mucosa intestinale, nei linfonodi e nell'endotelio. La sua prevalenza, probabilmente sottostimata, varia da 1 a 3 casi per milione di abitanti, quantomeno nella sua più grave manifestazione neonatale o infantile, nota anche come sindrome di Wolman. L'accumulo di esteri del colesterolo a livello epatico induce rapidamente steatosi microvescicolare con ipertransaminasemia ed epatomegalia, che evolve verso la cirrosi e l'insufficienza funzionale. L'ipercolesterolemia causa aterosclerosi precoce con elevata mobilità e mortalità per cause cardiovascolari. I bambini con LAL-D dimostrano crescita rallentata, diarrea, imponente epatosplenomegalia, anemia, insufficienza epatica e muoiono generalmente entro il primo anno di vita. Fino a pochissimi anni fa l'unica terapia possibile, a parte statine ed ezetimibe, consisteva nel trapianto di fegato o di cellule staminali ematopoietiche. Recentemente è stato introdotto nella pratica clinica il primo enzima ottenuto me-

\section{S. Cannavò \\ cannavos@unime.it}

1 Dipartimento di Patologia Umana dell'Adulto e dell'Età Evolutiva "G. Barresi”, Università di Messina, Messina, Italia diante tecniche di DNA ricombinante e dal 2013 sono stati pubblicati i primi studi di fase 2 e 3 , soprattutto condotti in pazienti adulti o comunque in bambini di età superiore ai 4 anni.

Lo studio di Jones et al. riporta i risultati del trattamento con sebelipase alfa in bambini di età inferiore a 6 mesi, con le manifestazioni più gravi della malattia e con la prognosi peggiore. Si tratta di uno studio multicentrico, aperto, di fase $2 / 3$ condotto in 9 differenti nazioni. I bambini sono stati trattati con sebelipase alfa a dose iniziale di $0,35 \mathrm{mg} / \mathrm{kg}$, incrementata progressivamente fino a $5 \mathrm{mg} / \mathrm{kg}$. Il principale obiettivo del trattamento era la sopravvivenza a 12 mesi dall'inizio del trattamento e oltre i 24 mesi di età.

Complessivamente sono stati trattati 9 bambini (età media 3 mesi). Il 67\% di essi sono sopravvissuti oltre i 12 mesi di età, mentre l'analisi retrospettiva di 21 neonati con la stessa malattia, che hanno costituito il gruppo di controllo, ha dimostrato una sopravvivenza a un anno di età pari allo $0 \%$. I bambini sopravvissuti fino al $12^{\circ}$ mese hanno dimostrato un evidente miglioramento della crescita ponderale e dell'anemia, un decremento degli indici di funzione epatica, una riduzione dell'epatosplenomegalia e dei sintomi gastroenterici. Tre bambini sono deceduti nei primi mesi di vita, mentre in un quarto bambino il decesso, avvenuto al $15^{\circ}$ mese di vita, è stato attribuito a cause non direttamente imputabili alla malattia principale. Gli altri 5 bambini sono sopravvissuti oltre i 24 mesi di età, dimostrando un evidente miglioramento dei parametri di crescita staturo-ponderale e degli indici di funzione epatica. In un solo caso sono stati evidenziati eventi avversi gravi durante l'infusione del farmaco (tachicardia, pallore, brividi e febbre).

In conclusione, il trattamento con sebelipase alfa si è dimostrato capace di migliorare la sopravvivenza e le condizioni cliniche di neonati con sindrome di Wolman, rara malattia causata da LAL-D. 